\title{
HIGH-RATE PHOTOMULTIPLIER-BASE AND PRECISE TIMING CALIBRATION
}

\author{
DANIEL BRAHY and EDOUARD ROSSA
}

CERN, Sile de Prévessin. F-01631 CERN Cedex, France

Received 27 June 1978

Four hodoscopes with 64 channels each have been designed to run in the high intensity muon beam of the SPS (up to $10^{9}$ muons $/ \mathrm{s}$ ). Despite a transmission at $310 \mathrm{~m}$ the double pulse resolution is $\leq 5 \mathrm{~ns}$ and a time jitter of about $100 \mathrm{ps}$ is achieved. The synchronisation is done with a sharp laser pulse.

\section{Introduction}

The photomultiplier-base described in this article belongs to a spectrometer which is placed in the high intensity muon beam. This spectrometer will allow the momentum of each beam particle ( $\left.10^{9} \mathrm{pps}\right)$ to be measured with a $1 \%$ accuracy and a dead time per channel smaller than 5 ns.

The necessary time resolution excludes the use of wire chambers as used in other beam spectrometers; here they are replaced by four hodoscopes.

In order to eliminate as many ambiguities as possible in the reconstruction of particle trajectories, their time of transit has to be measured with a resolution of $50 \mathrm{ps}$ and there must be the same order of stability for the 256 measuring channels.

A system of automatic calibration of the transit time has been installed for the whole detection and transmission chain which will meet these specifications.

This calibration is particularly necessary because the electronics (TDCs) for measuring the transit time are placed at a distance of up to $310 \mathrm{~m}$ from the hodoscopes (fig. 1).

\section{General description of the hodoscope}

Pilot U scintillator was selected (see ref. 1) and the light pipes are made of plexiglass 218 .

The photomultipliers used are the $9826 \mathrm{~B}$ from EMI, and more recently, the PM 1910 from RTC (13 $\mathrm{mm}$ photocathode diameter).

The scintillators are $20 \mathrm{~mm}$ thick in the direction traversed by particles. A height of $5 \mathrm{~mm}$, which is the same for all the fingers, allows a resolution of $\pm 0.5 \%$ on the measurement of the momentum. The length of the fingers has been chosen in order to have an intensity less than $3 \times 10^{7}$ pps on each photomultiplier (PM) when the total number of particles in the beam is $10^{9}$.

As the beam cross-section differs greatly for the four measuring planes, the four hodoscopes have different counter sizes, so as to distribute the intensity on the 64 PMs as evenly as possible. There

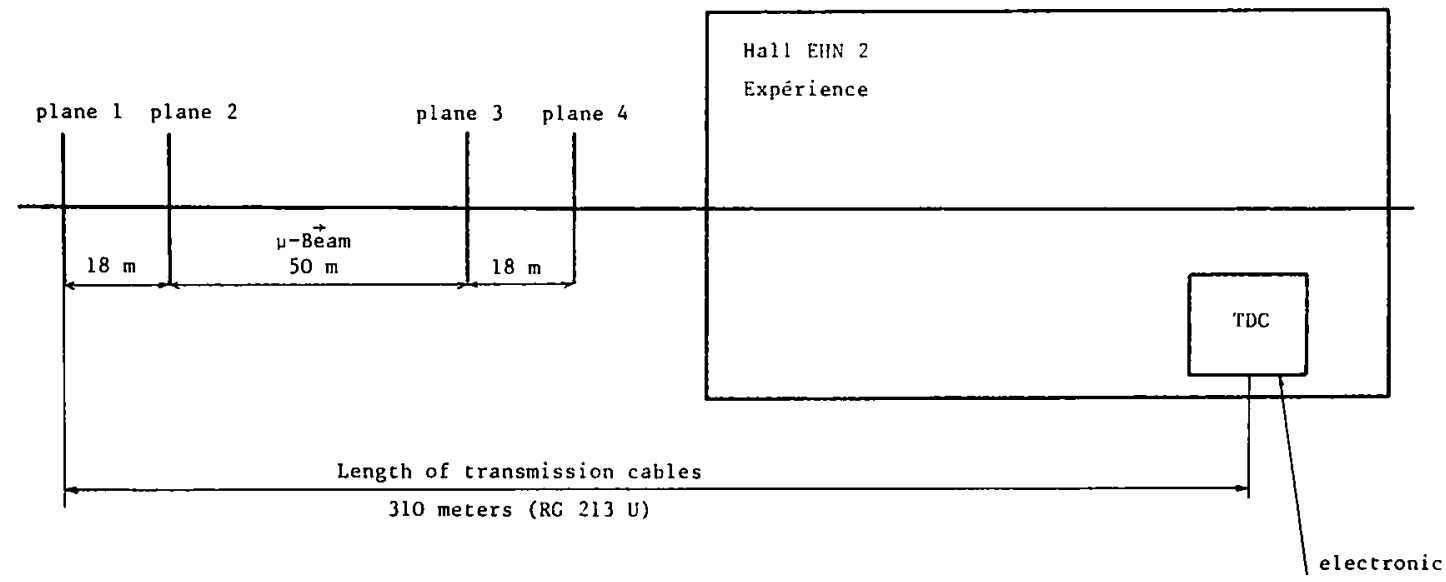

Fig. 1. General layout of the hodoscope. 


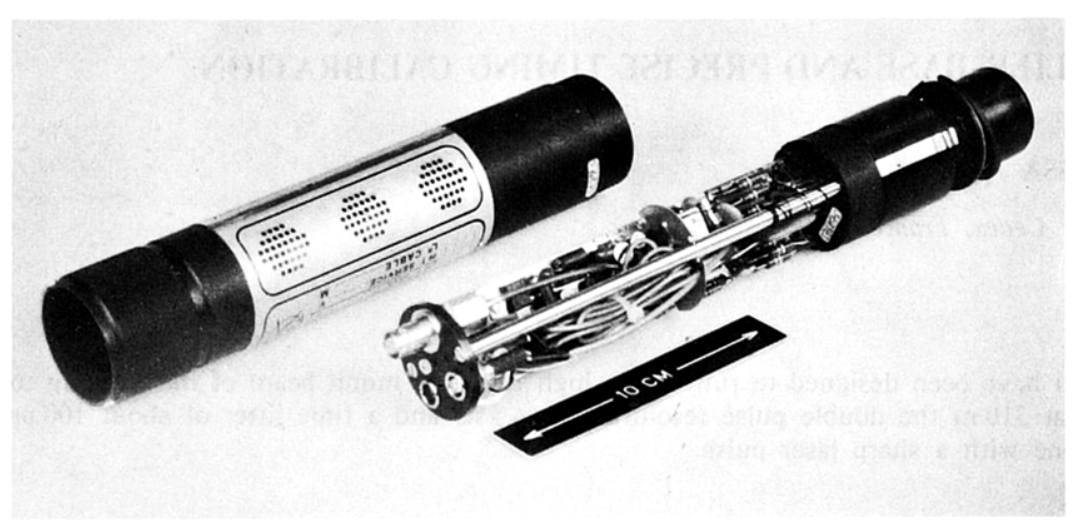

Fig. 2. Complete PM-base assembly open. It is possible to see the hv divider and alt the electronics.

is a few tenths of a millimeter overlap between the fingers.

\section{Photomultiplier-base (PM-base)}

(see figs. 2 and 3)

\subsection{AIM}

This base should comprise:

- a hv divider for supplying the photocathode and dynodes,

- a fast two-level discriminator capable of detecting the time of transit and accepting only pulses of high enough amplitude,

- a pulse forming circuit for the accepted pulses,

- a driving circuit for a long transmission line of $310 \mathrm{~m}$ long.

The total dispersion of this whole measuring chain (long cable included) must be less than $100 \mathrm{ps}$ even for two light pulses separated by $5 \mathrm{~ns}$.



Fig. 3. Complete PM-base. The left hand cover is to protect the photocathode when the PM is not plugged into the hodoscope.

\subsection{PM OPERATING CONDITIONS}

Many conflicting conditions must be satisfied.

On the one hand, the PM must tolerate $3 \times 10^{7}$ pulses $/ \mathrm{s}$ without exceeding the anode average current, which has been fixed by the manufacturer at $100 \mu \mathrm{A}$. On the other hand, the dispersion of the PM transit time decreases as the high tension increases. Similarly, the dispersion of the transit time $(\Delta t)$ decreases when the quantity of light received by the photocathode increases ${ }^{2}$ ) $\Delta t$ varies according to the formula:

\section{$\Delta t(\mathrm{fwhm})=k / \sqrt{ } n$,}

where $n$ is the number of photons. This has been verified in practice. In order to optimize the function of the PM, the quantity of light received by the photocathode was first increased by putting the maximum acceptable scintillator thickness in the beam, that is to say $20 \mathrm{~mm}$.

Secondly, the dispersion of the transit time has been reduced by setting the maximum permissible voltage between the photocathode and the first dynode, and thereafter between each dynode.

In these conditions, the current on the anode would be considerable, well above $100 \mu \mathrm{A}$. To avoid exceeding this limit, the first dynode which reached a current of $100 \mu \mathrm{A}$ was used as the output anode. A peak current of $800 \mu \mathrm{A}$ for light pulses produced by particles crossing $20 \mathrm{~mm}$ of pilot $U$, was set as a limit.

The average current for $3 \times 10^{7}$ pulses/s is $72 \mu \mathrm{A}$ assuming triangular pulses of $6 \mathrm{~ns}$ at the base. Taking into account the duty cycle of the SPS (cycle time of 6-10 s), this gives a good safety margin, since the recommendation of the PM manufacturers is $100 \mu \mathrm{A}$ average anode current.

In these working conditions, we get a peak cur- 


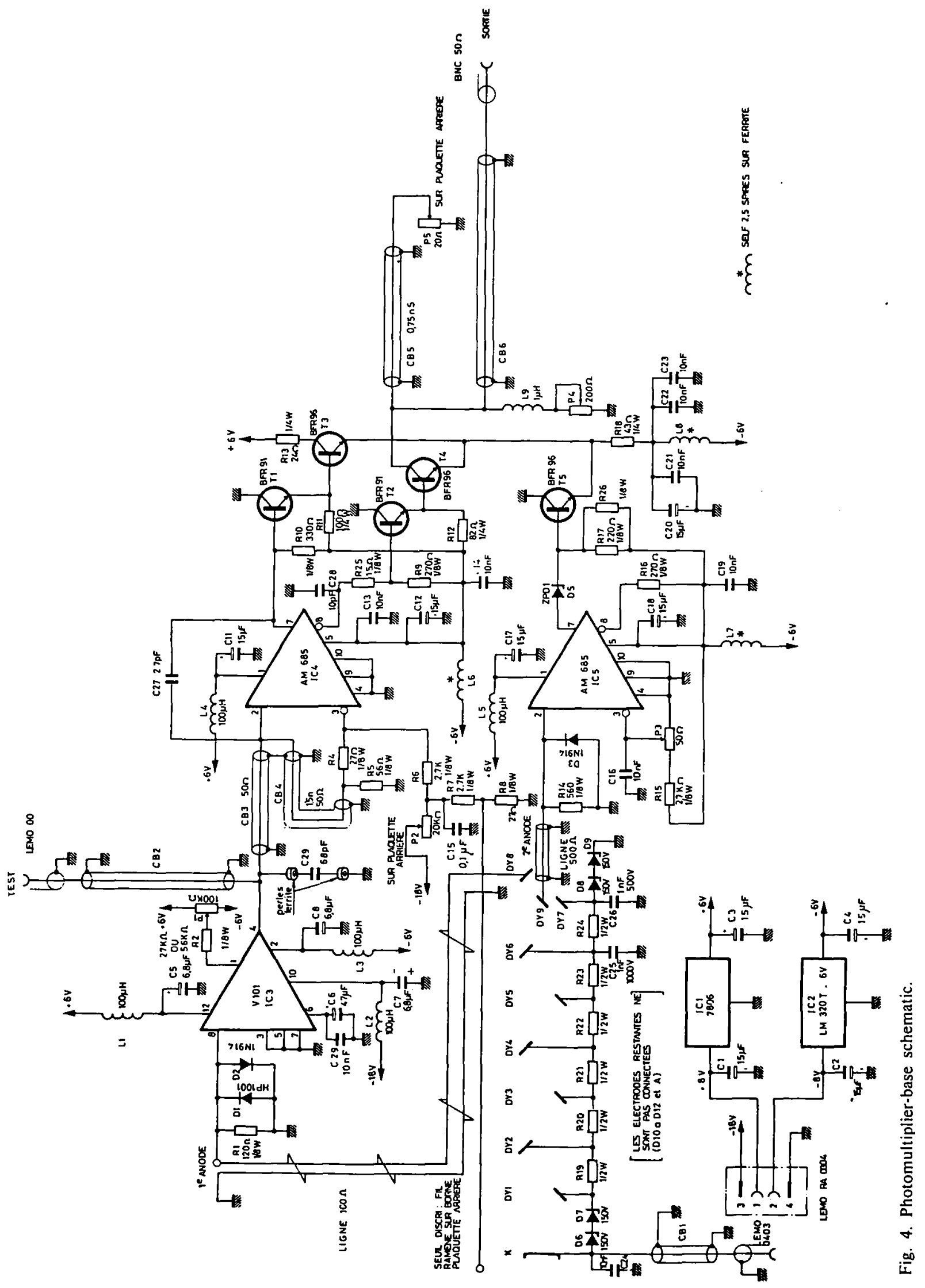


rent of $800 \mu \mathrm{A}$ on the sixth, seventh or eighth $\mathrm{dy}$ node depending on individual photomultiplier sensitivity.

\section{Circuit description}

\subsection{HV DIVIDER}

The circuit diagram of the base is given on the opposite page, for the PM9826 (see fig. 4).

The dynode number 8 is the signal output of the PM. Dynode 9 gives a current of about $1 / 3$ of the current of the preceding dynode despite their both being at earth potential. It collects part of the secondary emission of the dynode number 8 .

For the PM 1910 RTC the dynode number 8 always gives the signal for the V 101 amplifier.

The gate signal is always picked-up on the grid type anode.

The total high voltage is of the order of $1800 \mathrm{~V}$. The current rise time for dynode number 8 is about $1.5 \mathrm{~ns}$. The pulse width at half-height is $<3$ ns with $50 \Omega$ termination. The transit time of the PM tube is less than $15 \mathrm{~ns}$.

\subsection{Two-LEVEL DISCRIMINATION}

In fig. 4 one can see that dynode number 9 acts as a second anode and allows the opening of an 8 ns wide gate if the current amplitude is sufficient.

The charge resistance of this second anode is large enough to broaden the pulse.

The comparator AM 685 (IC5) controls transistor T5 which acts as a gate for the output pulses.

Dynode number 8 acts as a first anode in order to measure accurately the arrival time of the pulse (fig. 4).

Here we must detect the passage of the current to a level equal to $1 / 10$ of the peak, that is to say $80 \mu \mathrm{A}$. The hybrid amplifier $\mathrm{V} 101$ has a voltage gain of ten, and drives the first comparator AM 685 (IC4). The threshold of this comparator has been adjusted to $100 \mathrm{mV}$. But the threshold of the gate comparator (IC5) is adjusted at half of the nominal light amplitude. The comparator (IC4) is used in a differential mode, and looks at the voltage change in $1.5 \mathrm{~ns}$ (CB4 delay time). This gives the same result as a clipping line but without the drawback of multiple reflections.

The main advantages are:

- the possibility of separating pulses with a large pile-up,

- the output zero offset drift of the V 101 has no effect on the real threshold,
- the noise at lowest frequencies is not detected. For example the $50 \mathrm{~Hz}$ has no effect until it does not sature the V 101 amplifier.

\subsection{Driving Circuit for the transmission CABLE}

This circuit consists of five transistors which act as a logic gate and as a cable driver. To avoid piling up of pulses along the transmission cable, it is necessary to send a symmetrical signal for each accepted pulse from the photomultiplier. This is achieved by a short clipping cable in parallel with the output (CB5).

The gate acts as a current switch and the speed of the AM 685 comparators is matched by the transistors BFR 96 and BFR 91 which have a cutoff frequency $f i$ of $5 \mathrm{GHz}$.

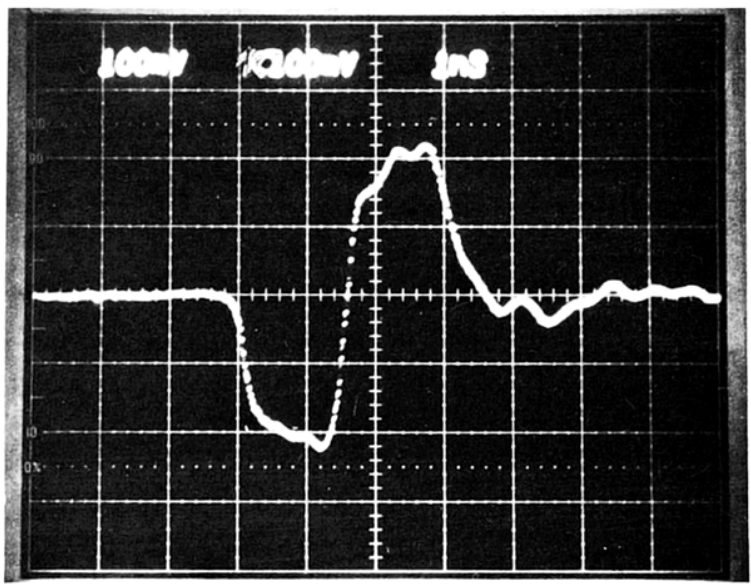

Fig. 5. Output pulse of PM-base: vert. scale: $1 \mathrm{~V} /$ division. hor. scale: I ns/division.

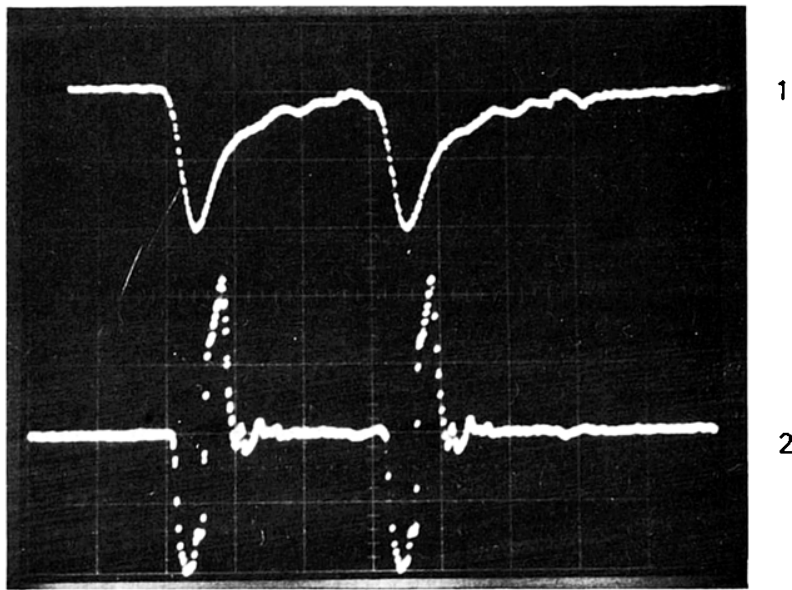

Fig. 6. Input pulses of the base (simulation of light pulses by integration of short pulses with $200 \mathrm{~m}$ of cable RC 213), lowes trace is the output pulse of PM-base. Scale: $V_{1}=50 \mathrm{mV} /$ division, $\mathrm{V}_{2}=1 \mathrm{~V} /$ division, $\mathrm{H}=5 \mathrm{~ns} /$ division. 
Without any clipping, the output pulse has a width of 2 ns at the base and an amplitude of $2 \mathrm{~V}$.

The rise and fall times (10-90\%) are less than $0.5 \mathrm{~ns}$.

The amplitude of these signals is sufficient to drive NIM electronics directly.

The clipping cable (CB5) is very short (0.75 ns) and makes the output pulse symmetrical. The base output pulse shape terminated by $50 \Omega$ is shown in figs. 5 and 6 . After $310 \mathrm{~m}$ of cable RG 213 the pulses are shown in fig. 7 .

\section{Adjustments}

Potentiometers: P1 fixes the zero offset of amplifier V 101;

P2 fixes the low level threshold (1/10 of signal of $\mathrm{V} \mathrm{101);}$

P3 fixes high level gate threshold;

P4 and P5 match the driver with different lengths of transmission cable.

\subsection{Transmission Cable}

We have chosen the RG $213 \mathrm{U}$ cable, as it shows an acceptable attenuation. After $310 \mathrm{~m}$ cable length the output pulse has a $6 \mathrm{~ns}$ width at the base and a $150 \mathrm{mV}$ height, fwhm $\simeq 2 \mathrm{~ns}$.

The amplitude is sufficient to trigger the NIM discriminators.

\subsection{RESULTS OBTAINED}

The possibility of separating very close pulses is slightly diminished by the transmission cable. But,

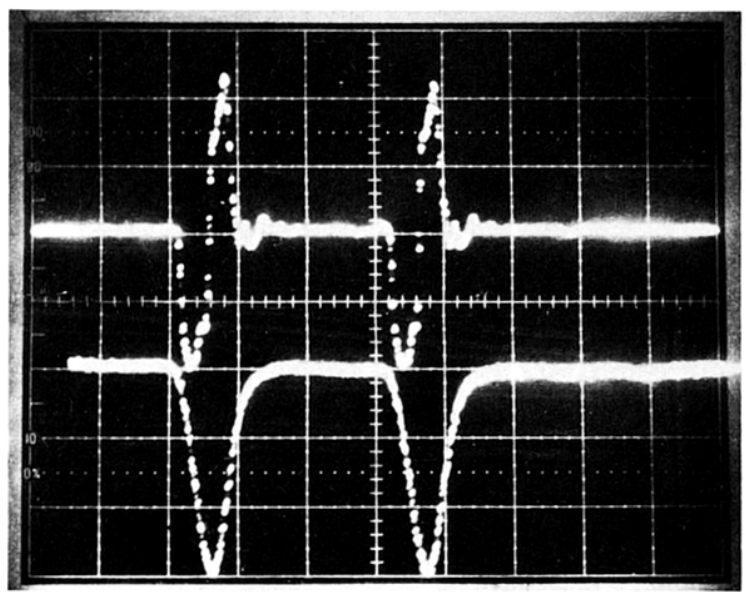

Fig. 7. Output pulses of PM-base, scale: $1 \mathrm{~V} /$ division. Output pulses after $310 \mathrm{~m}$ of RG 213 cable, scale: $50 \mathrm{mV} /$ division (vert.), $5 \mathrm{~ns}$ /division (hor.).

due to the cable driving circuit one may separate pulses $5 \mathrm{~ns}$ apart without problems. The limit of separation at the base of the pulses is about $5 \mathrm{~ns}$ after $310 \mathrm{~m}$ of cable RG 213 (see fig. $8 \mathrm{a}, \mathrm{b}$ ).

Fig. 8 shows the double pulse separation of the whole system. There is no appreciable pile up effect. The digital separation of the whole circuit, cable included, is above $200 \mathrm{MHz}$. If, for instance, one wished to count the pulses and a relatively short transmission cable $\simeq 166 \mathrm{~m}$ was used, the double pulse resolution is $3.5 \mathrm{~ns}$ (see fig. 9). With the beam, the measurement of the spread of the transit times between two channels in the hodoscope has shown fwhm 350 ps (including $310 \mathrm{~m}$ of cable). The spread of the transit times for each single channel is 250 ps fwhm.
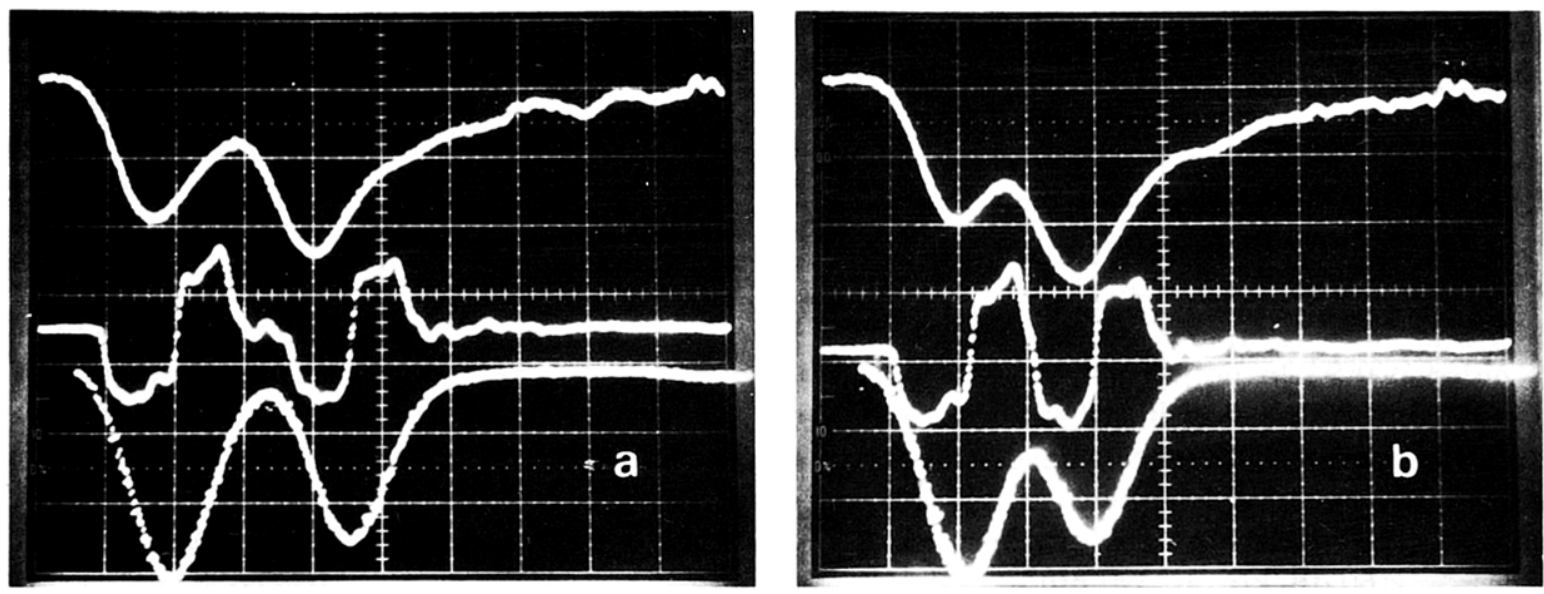

Fig. 8. (a) Double pulse resolution of channels with $310 \mathrm{~m}$ of cable. Upper signal: simulated light pulses with 5 ns separation. Center signal: output of PM-base. Lower signal: output after $310 \mathrm{~m}$ of cable RG 213. (b) Double pulse resolution of channels with $310 \mathrm{~m}$ of cable but $4 \mathrm{~ns}$ between input pulses. 


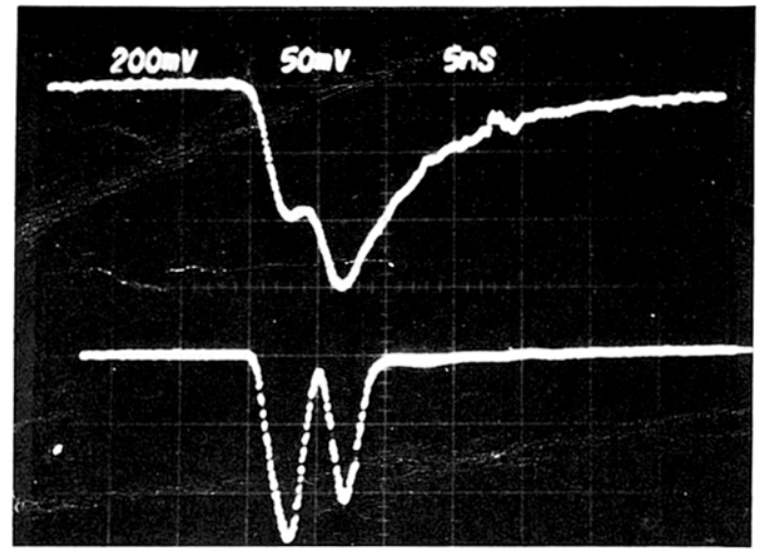

Fig. 9. Double pulse resolution with $166 \mathrm{~m}$ of transmission cable RG 213. Scale: upper trace $50 \mathrm{mV} /$ division input signal of PM-base, lower trace output of transmission cable. $200 \mathrm{mV} /$ division.

\section{Calibration}

\subsection{LiGHT SOURCE}

The calibration of the 256 channels will be performed by means of light pulses distributed to each photomultiplier.

One light pulse with fast rise times is sent simultaneously to the $256 \mathrm{PMs}$ of all four hodoscopes through fiber optics of $2 / 10 \mathrm{~mm}$ diameter. The source of the light is a nitrogen UV laser. This laser emits light at a wavelength of $337 \mathrm{~nm}$ and excites a cell containing POPOP* dissolved in toluene. The cell lases at about $430 \mathrm{~nm}$ and produces an intense blue light.

A fast detector placed at the output of the laser generates the "Start" pulse. The "Stops" come from the PM of each measuring channel.

\subsection{LASER'S MAIN CHARACTERISTICS}

Type SOPRA;

$\lambda=337 \mathrm{~nm}$;

pulse width at half maximum output: $2.4 \mathrm{~ns}$; peak light power: $400 \mathrm{~kW}$;

external trigger.

POPOP-toluene dye: $\lambda \simeq 430 \mathrm{~nm}$, $\mathrm{fwhm} \simeq \mathrm{l} \mathrm{ns}$.

\subsection{MAIN CHARACTERISTICS OF THE FIBERS}

These are fibers which have been developed for data communications (type FORT).

Attenuation: $\lesssim 20 \mathrm{~dB} / \mathrm{km}$ at $850 \mathrm{~nm}$, $<60 \mathrm{~dB} / \mathrm{km}$ at $390 \mathrm{~nm}$;

Useful section: $0.2 \mathrm{~mm}$;

* p-bis [2-(5-phenyloxazolyl)] benzene.

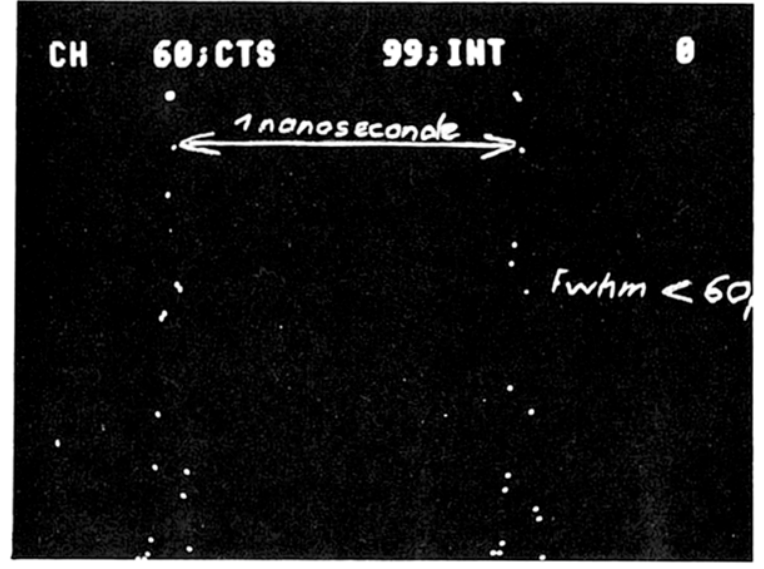

Fig. 10. Time dispersion between start pulse coming from the laser and measuring channel of the hodoscope. The two hislograms are separated by changing the delay line start by 1 ns: this gives a horizontal scale calibration of $8.8 \mathrm{ps}$ per channel. Time dispersion is $\mathrm{fwhm}<60$ ps.

resistance to traction: $>6 \mathrm{daN}$;

maximum length of fiber used: $76 \mathrm{~m}$.

\subsection{RESULTS OBTAINED}

The time dispersion between the photodiode at the output of the laser and the output pulses of photomultipliers was measured, operating under the conditions described in section 3 . They were illuminated by optic fibers $20-70 \mathrm{~m}$ long coming from a POPOP-toluene dye cell excited by the laser.

The measured dispersion was less than 100 ps at half height for all channels.

Many of them give 60 ps fwhm. The resolution of measurement was $8.8 \mathrm{ps} /$ channel (see fig. 10).

\section{Conclusion}

Results obtained with the beam:

The time jitter per channel, transmission cable up to $310 \mathrm{~m}$ included, is $250 \mathrm{ps}$ fwhm. The double pulse separation is $5 \mathrm{~ns}$.

Results of calibration with laser:

The dispersion per channel is $100 \mathrm{ps}$ fwhm.

We wish to thank C. Bovet and the members of his group for the help during the development of the hodoscopes, in particular R. Maleyran for the design of the mechanics.

We also acknowledge F. Navach (Group NA 4) for his help in the tests of the PM base.

\section{References}

1) D. Brahy and E. Rossa, SPS/EBP/Note 77.5 (15.3.1977).

2) Photomultiplier Manual RCA (1970). 
NUCLEAR INSTRUMENTS AND METHODS 158 (1979) 127-128; (C) NORTH-HOLLAND PUBLISHING CO.

\title{
ESTIMATING THE ERROR IN THE INVERSE MOMENTUM FOR PARTICLE TRACKS IN HIGH ENERGY PHYSICS USING WIND'S QUINTIC SPLINE METHOD
}

\author{
HENDRIK CORPORAAL \\ CERN, Geneva, Switzerland
}

Received 22 August 1978

An expression for the inverse momentum is differentiated with respect to the observations with the object of evaluating an error estimate in terms of the uncertainty in the observations.

In high energy physics experiments the momenta of particles are measured by observing their trajectories through magnetic spectrometers. In ref. 1 it is shown for such a track that

$p y^{\prime \prime}=\left(1+y^{\prime 2}+z^{\prime 2}\right)^{\frac{1}{2}}\left[B_{x} z^{\prime}+B_{y} y^{\prime} z^{\prime}-B_{z}\left(1+y^{\prime 2}\right)\right]$,

$p z^{\prime \prime}=\left(1+y^{\prime 2}+z^{\prime 2}\right)^{ \pm}\left[B_{x} y^{\prime}-B_{z} y^{\prime} z^{\prime}+B_{y}\left(1+z^{\prime 2}\right)\right]$,

where $y$ and $z$ are coordinates perpendicular to the $x$-axis of the spectrometer, $y^{\prime \prime}$ denotes $\mathrm{d}^{2} y / \mathrm{d} x^{2}$, etc., $p$ the momentum in normalised units, and $B_{x}, B_{y}, B_{z}$ are the magnetic field components, which will be functions of $x, y$ and $z$.

There are $N$ detectors measuring the $y_{i}$ and $z_{i}$ coordinates of the particle track at $x_{i}$. If the righthand sides of eqs. (1) were known, they could be integrated twice giving two functions $Y(x)$ and $Z(x)$ with boundary conditions $Y(x)=0$, $\mathrm{d} Y(x) / \mathrm{d} x=0$ at $x=x_{1}$, and similarly for $Z(x)$. If there are measurements of $y_{i}, z_{i}$ at enough points $x_{i}$, the right-hand sides can be estimated everywhere using, e.g., a magnetic field table for $\boldsymbol{B}(x, y, z)$ and an appropriate interpolation procedure for the $y, z$ coordinates. Ref. 1 shows how this can be done using a cubic spline approximation; another procedure is given in ref. 2.

Assuming $p$ to be constant, the track is written as

$y(x)=a_{1}+a_{2}+\frac{1}{p} Y(x)$,

$z(x)=b_{1}+b_{2}+\frac{1}{p} Z(x)$,

where $a_{1}, a_{2}, b_{1}$ and $b_{2}$ are the as yet unknown integration constants.

It is then possible to make a least-squares fit to eqs. (2) for $a_{1}, a_{2}, b_{1}, b_{2}$ and $1 / p$, the left-hand sides being the observed track coordinates $y_{i}$ and $z_{i}$. This results in five linear equations. Solving these equations one gets for $1 / p^{2.3}$ )

$$
\begin{aligned}
& T_{3}\left[\left(y_{i} Y_{i}\right)+\left(z_{i} Z_{i}\right)\right]-T_{1}\left(Y_{i}\right)-T_{4}\left(x_{i} Y_{i}\right)- \\
& \frac{1}{p}=\frac{-T_{6}\left(Z_{i}\right)-T_{8}\left(x_{i} Z_{i}\right)}{T_{3}\left[\left(Y_{i}^{2}\right)+\left(Z_{i}^{2}\right)\right]-T_{2}\left(Y_{i}\right)-T_{5}\left(x_{i} Y_{i}\right)-}, \\
& -T_{7}\left(Z_{i}\right)-T_{9}\left(x_{i} Z_{i}\right)
\end{aligned}
$$

where

$$
\begin{aligned}
& T_{1}=\left(x_{i}^{2}\right)\left(y_{i}\right)-\left(x_{i}\right)\left(x_{i} y_{i}\right), \\
& T_{2}=\left(Y_{i}\right)\left(x_{i}^{2}\right)-\left(x_{i} Y_{i}\right)\left(x_{i}\right), \\
& T_{3}=N\left(x_{i}^{2}\right)-\left(x_{i}\right)^{2}, \\
& T_{4}=-\left(x_{i}\right)\left(y_{i}\right)+N\left(x_{i} y_{i}\right), \\
& T_{5}=-\left(Y_{i}\right)\left(x_{i}\right)+N\left(x_{i} Y_{i}\right), \\
& T_{6}=\left(x_{i}^{2}\right)\left(z_{i}\right)-\left(x_{i}\right)\left(x_{i} z_{i}\right), \\
& T_{7}=\left(x_{i}^{2}\right)\left(Z_{i}\right)-\left(x_{i}\right)\left(x_{i} Z_{i}\right), \\
& T_{8}=-\left(x_{i}\right)\left(z_{i}\right)+N\left(x_{i} z_{i}\right), \\
& T_{9}=-\left(Z_{i}\right)\left(x_{i}\right)+N\left(x_{i} Z_{i}\right) .
\end{aligned}
$$

A $\sum_{i=1}^{N}$ summation has been omitted before every left-hand parenthesis, (.

We now want to make an estimate of the error we can expect in $1 / p$ when we assume random and uncorrelated errors in the observations $y_{j}$ and $z_{i}$.

We assume no variations in $Y_{j}$ and $Z_{j}$. This is justified if the variation of $\int B \mathrm{~d} l(l$ being the path of the particle) can be neglected for small variations in $y_{j}$ and $z_{j}$. The detector positions $x_{j}$ are also fixed. To evaluate the error in $1 / p$ we now have to determine the derivatives

$\frac{\partial(1 / p)}{\partial y_{j}}$ and $\frac{\partial(1 / p)}{\partial z_{j}}$, for $j=1$ to $N$.

We note that of the terms $T_{1}, T_{2}, T_{3}, \ldots, T_{9}$ only 
$T_{1}$ and $T_{4}$ depend on $y_{j}$.

and only

$T_{6}$ and $T_{8}$ depend on $z_{j}$.

In particular the denominator of eq. (3), let us call it $D$, depends neither on $y_{\mathrm{j}}$ nor on $z_{j}$.

From eqs. (4) we find

$\frac{\partial T_{1}}{\partial y_{j}}=\frac{\partial T_{6}}{\partial z_{j}}=\left(x_{i}^{2}\right)-\left(x_{i}\right) x_{j}$,

and

$\frac{\partial T_{4}}{\partial y_{j}}=\frac{\partial T_{8}}{\partial z_{j}}=N x_{j}-\left(x_{i}\right)$,

with the same summation convention.

We then find

$$
\begin{aligned}
\frac{\partial(1 / p)}{\partial y_{j}}= & \left\{T_{3} Y_{j}-\left(Y_{i}\right)\left[\left(x_{i}^{2}\right)-\left(x_{i}\right) x_{j}\right]-\right. \\
& \left.-\left(x_{i} Y_{i}\right)\left[N x_{j}-\left(x_{i}\right)\right]\right\} / D \\
= & \left\{T_{3} Y_{j}-x_{j} T_{5}-T_{2}\right\} / D,
\end{aligned}
$$

and

$$
\begin{aligned}
\frac{\partial(1 / p)}{\partial z_{j}}= & \left\{T_{3} Z_{j}-\left(Z_{i}\right)\left[\left(x_{i}^{2}\right)-\left(x_{i}\right) x_{j}\right]-\right. \\
& \left.-\left(x_{i} Z_{i}\right)\left[N x_{j}-\left(x_{i}\right)\right]\right\} / D \\
= & \left\{T_{3} Z_{j}-x_{j} T_{9}-T_{7}\right\} / D,
\end{aligned}
$$

where the second and fourth line are obtained by using eqs. (4) again.

The square of the expected error in $1 / p$ thus becomes

$$
\begin{aligned}
\left(\Delta \frac{1}{p}\right)^{2}= & \sum_{j=1}^{N}\left[\left(T_{3} Y_{j}-x_{j} T_{5}-T_{2}\right)^{2} \Delta y_{j}^{2}+\right. \\
& \left.+\left(T_{3} Z_{j}-x_{j} T_{9}-T_{7}\right)^{2} \Delta z_{j}^{2}\right] / D^{2},
\end{aligned}
$$

where $\Delta y_{j}$ and $\Delta z_{j}$ are the expected errors in $y_{j}$ and $z_{j}$ respectively.

We have checked this formula numerically on IBM370, using double precision. By introducing deviations in each of the coordinates eq. (5) was verified.

The author is indebted to $\mathrm{D}$. Townsend and to his supervisor $\mathrm{H}$. Wind for valuable discussions.

\section{References}

1) H. Wind, Nucl. Instr. and Meth. 115 (1974) 431.

2) H. Wind, Nucl. Instr. and Meth. 153 (1978) 195.

3) D. W. Townsend and J. D. Wilson, CERN/DD/75/2, private communication. 